Check for updates

Cite this: RSC Adv., 2019, 9, 29805

Received 6th July 2019

Accepted 16th September 2019

DOI: $10.1039 / c 9 r a 05125 b$

rsc.li/rsc-advances

\section{An ultrasensitive Fano resonance biosensor using two dimensional hexagonal boron nitride nanosheets: theoretical analysis $\dagger$}

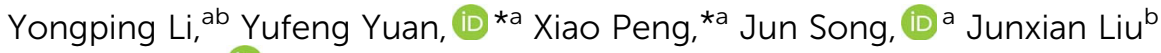 \\ and Junle Qu iD a
}

\begin{abstract}
This study proposed a novel Fano resonance (FR) biosensor with ultrahigh detection sensitivity by integrating two dimensional (2D) hexagonal boron nitride (h-BN) nanosheets with a plasmonic silver film-silicon hybrid nanostructure. Owing to its ultralow-loss in surface plasmon polaritons (SPPs), 2D hBN nanosheets can act as a planar photon waveguide (PWG) for generating energy level splitting. Notably, both asymmetric FR sharp lines and plasmon induced transparency (PIT) can be produced by modulating the coupling strength between the planar PWG mode provided by h-BN nanosheets and the surface plasmon polariton (SPP) mode in the silver film-silicon hybrid nanostructure. Compared with conventional phase-modulation SPR biosensors, our proposed configuration based on Fano resonance can produce ultrahigh reflectivity of 0.934 and overcome the limitation of quasi-darkness reflectivity which is difficult for further phase extraction. More importantly, our proposed FR configuration can provide a promising phase detection sensitivity as high as $3.13 \times 10^{6}$ degree per RIU (refractive index unit, RIU), which is enhanced by almost 100 times compared with conventional phase-modulation SPR biosensors. In addition, our proposed configuration has also shown the characteristics of multiple-order Fano resonances, largely depending on the partial coupling between the SPP mode and the differentorder PWG mode. Our proposed FR biosensor can provide a highly promising candidate for designing a multiple-order FR platform for performing ultrasensitive detection.
\end{abstract}

\section{Introduction}

Optical surface plasmon resonance (SPR) biosensors designed for monitoring targeted biomolecules exhibit well-accepted features, such as high sensitivity, high speed, and label-free quantitation..$^{1-3}$ To achieve attenuated total reflection (ATR), conventional SPR biosensors usually utilize Kretschmann or Otto coupling configurations. ${ }^{1}$ In the conventional SPR configuration, except for a high refractive index prism, a metallic thin layer such as gold, or silver, and aluminum, is employed to provide surface plasmon polariton (SPP) waves, which can be resonant with incident photons. When the obtained reflectivity in a SPR curve has a darkness or quasi-darkness point, it shows that almost all the incident photons $(\approx 100 \%)$ have been absorbed and transferred into energy to support the resonance

${ }^{a}$ Key Laboratory of Optoelectronic Devices and Systems of Ministry of Education and Guangdong Province, College of Physics and Optoelectronic Engineering, Shenzhen University, Shenzhen, 518060, People's Republic of China. E-mail: yfyuan@szu.edu. cn; pengxiao_px@szu.edu.cn

${ }^{b}$ College of Physical Science and Technology, Guangxi Normal University, Guilin, 541004, People's Republic of China

$\dagger$ Electronic supplementary information (ESI) available. See DOI: 10.1039/c9ra05125b of SPPs waves. It is well-known that, the high sensitivity and resolution are two critical parameters to judge a high quality SPR sensor. However, both the sensitivity and resolution of metal-based SPR biosensors are largely limited, due to the poor photon absorption efficiency of bared metal film and broad full width at half maximum (FWHM) from SPR curves. Moreover, it is still highly desirable to develop more novel SPR biosensors for quantitatively monitoring the target analytes with low molecular weight (smaller than a few hundreds of Daltons) in ultralow detection concentrations. To solve the challenges, it is of considerable significance to develop novel optical SPR biosensors with ultrahigh sensitivity and resolution for meeting practical applications.

In addition to the wavelength, amplitude, and polarization, phase is also another important parameter that extracts optical property of reflected light. To further develop detection sensitivity, phase modulation-based SPR sensors have been proposed. ${ }^{4,5}$ Prior to phase determination, it needs to fix both the incident light and wavelength. For phase-modulation SPR, two interferential light beams including p-polarized light and spolarized light have a significant differential phase. Thus, the differential phase can be considered as an index to evaluate SPR performance. It has been confirmed that, phase modulation is more sensitive than angle, amplitude or wavelength 
modulation..$^{6-9}$ The reason is that, the phase jump at the SPR angle is highly related with the minimum reflectivity. Under the ATR, the strongest phase jump usually occurs at the typical dip of smallest minimum reflectivity, which corresponds to the strongest SPR enhancement. ${ }^{10}$ However, the largest differential phase always corresponds to a darkness or quasi-darkness point, resulting in an extremely weak p-polarized reflection light. Although phase-modulation SPR sensors have high sensitivity, they largely suffer from weak intensity of reflection light, because it needs to measure the intensity of reflected light to calculate the differential phase. ${ }^{11}$ Moreover, phasemodulation approach cannot significantly sharpen the SPR curve and further develop the detection resolution. Thus, it is more preferable to obtain a p-polarized reflected light with high intensity and narrow SPR reflection.

It is worth noting that, hybrid strong coupling from different electromagnetic (EM) states is a promising approach to produce a narrow energy level splitting. ${ }^{12}$ As a typical example of plasmon resonance in photonics, Fano resonance can be realized by the strong interference between a discrete quantum state and a continuum band of states, resulting in a plasmon transparent narrow window in a broad absorption band..$^{13}$ In addition, Fano resonance (FR) widely exists in a host of natural and artificial configurations. ${ }^{14}$ Owing to its unique sharp resonance, plasmon induced transparency (PIT) has shown great potential in exploiting high quality SPR sensors. For example, for a planar multilayer configuration including surface plasmon polaritons (SPPs) mode and photonic waveguide mode, FR can be generated, forming a narrow optical transparent window. Conventional SPR sensors usually have a large energy loss, and provide a sensitivity enhancement by largely absorbing photon energy to produce enhanced electric field. However, FR-based sensors can produce dark states, and it needn't radiate energy outward. Thus, Fano resonance can provide a larger sensitivity enhancement than conventional SPR. For theoretical proposals, Hayashi et al. ${ }^{\mathbf{1 5 , 1 6}}$ proposed a waveguide-coupled SPR sensor based on SPPs and waveguide modes, which produce sharp Fano resonance line, which can be employed to perform highresolution sensing. In addition, Huang et al. ${ }^{17}$ proposed an ultrasensitive FR biosensor working in near-infrared region. In the proposed configuration, indium tin oxide layer (ITO) is plasmonic materials that support SPPs mode, while a silicon layer provides photonic PWG mode. For experimental observations, Recently, Zheng et al. ${ }^{18}$ developed a FR-enhanced SPR sensor, providing an intensity sensitivity enhancement of 356 times higher than conventional SPR sensors. Also, they observed Fano resonance lines in 2D materials (graphene$\mathrm{MoS}_{2}$ )-related hybrid nanostructures. More recently, Ruan et $a l .{ }^{19}$ proposed a multilayer structure employing two planar waveguides (PWGs) and few layered graphene. Owing to the low losses of surface plasmon polaritons from graphene layers, the Fano resonance lines originate from the different quality factors in two waveguides. Current observations have shown that, although employing 2D materials can develop the detection sensitivity, the introduction of $2 \mathrm{D}$ material layers is not the most pivotal factor to generate Fano resonance. However, it can be included that, instead of conventional metal materials, employing ultralow-loss of SPPs material for designing FRbased photonic device is a promising option.

Fortunately, 2D hexagonal boron nitride (h-BN), which is known as white graphene, ${ }^{\mathbf{2 0 , 2 1}}$ has shown great potential in exploiting Fano resonance-enhanced sensors, due to its ultralowloss of surface plasmon polaritons, ${ }^{22,23}$ excellent plasmonic enhancement, ${ }^{24}$ and ultrahigh crystal stability. ${ }^{25-27}$ In addition, the h-BN nanosheets have not only excellent reliability and compatibility with other materials, ${ }^{28}$ but also can capture aromatic biochemical molecules by dipole-dipole adsorption force. ${ }^{24}$ Moreover, the h-BN nanosheets can be employed as planar photon waveguide for controlling and manipulating light. ${ }^{29-32}$ It is worth noting that, h-BN is intrinsically anisotropic, while the averaged isotropic dielectric constant of h-BN nanosheets with a thickness up to several hundred nanometers can be measured by optical approaches. ${ }^{27,33}$ Unlike other 2D materials (such as graphene, transition metal dichalcogenides, MXenes, black phosphorous, and antimonene, etc.), h-BN has a negligible imaginary part of dielectric constant, indicating that ultralow-loss of polaritons is possible for h-BN. Inspired by these observations, we theoretically proposed a novel ultrasensitive FR-enhanced biosensor consisting of plasmonic silver film, $\mathrm{SiO}_{2}$ interlayer, and 2D material h-BN layer. It can be found that, the proposed configuration can produce multiple-order asymmetric FR lines by modulating the coupling strength between the PWG mode from the h-BN nanosheets and SPPs mode from plasmonic silver film-silicon hybrid nanostructure in visible region. Compared with conventional SPR sensor, our proposed biosensor designed by phase modulation, can provide a phase detection sensitivity as high as $3.13 \times 10^{6}$ degree per RIU, which is enhanced by almost 100 times. More

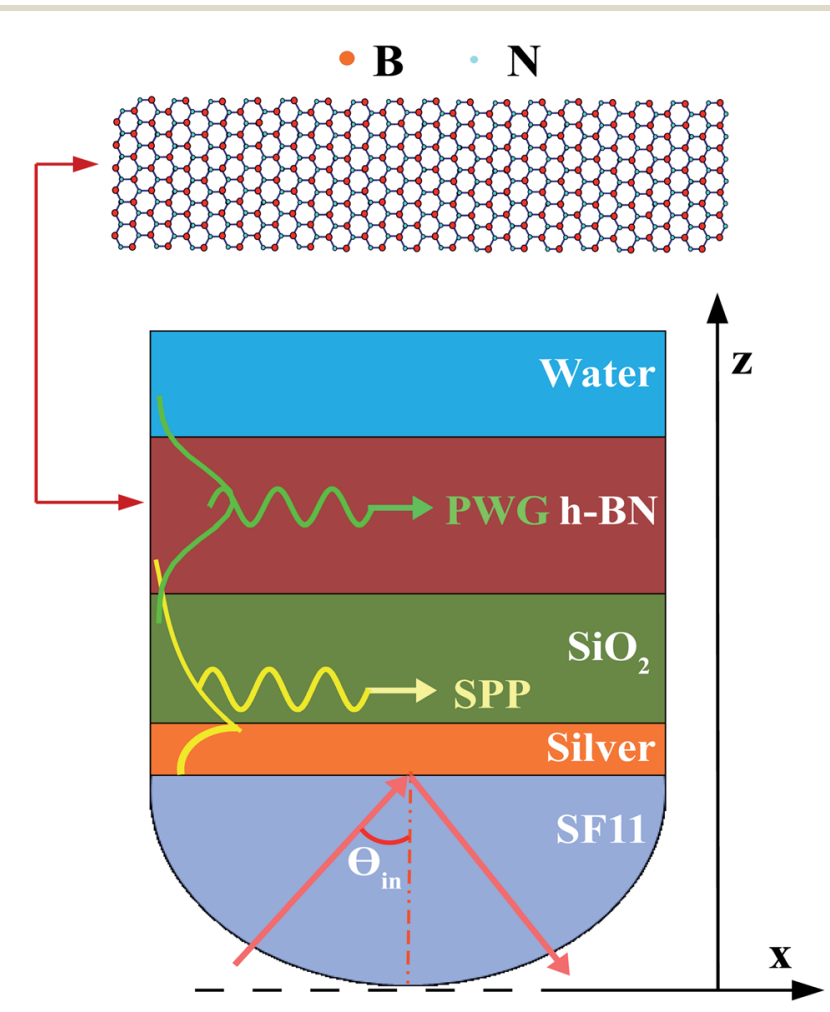

Fig. 1 Schematic of FR-based hybrid configuration. 
importantly, it is the first demonstration of FR biosensor with multiple-order features created by employing promising 2D material in visible region.

\section{Theoretical model and design consideration}

A classical Kretschmann configuration consists of a SF11 prism, $\mathrm{Ag}$ thin film, $\mathrm{SiO}_{2}$ interlayer, h-BN layer, and sensing medium (water), as shown in Fig. 1. In our proposed hybrid configuration, all the stacked layers are considered optically isotropic and non-magnetic. In addition, the $\mathrm{Ag}-\mathrm{SiO}_{2}$ hybrid structure can provide the SPPs mode, which stands for a bright mode having a broad resonance, while the h-BN nanosheet can provide PWG mode, which denotes a dark mode with a sharp resonance. Under the excitation of a p-polarized (TM) incident light at $632.8 \mathrm{~nm}$, the SPPs mode can be excited and propagated along the $\mathrm{Ag}-\mathrm{SiO}_{2}$ interface. Also, the PWG mode can be formed within the $\mathrm{SiO}_{2}-\mathrm{h}-\mathrm{BN}$ hybrid nanostructure. Once the tail of SPPs evanescent filed overlaps one of the PWG modes, the two modes can interact each other, ultimately generating narrow FR lines. Finally, the coupling strength and FR order can be modulated by varying the thickness of $\mathrm{SiO}_{2}$ interlayer, and h$\mathrm{BN}$, respectively.

The refractive indices of stacked components in our proposed configuration are critical to calculate the FR reflectivity and detection sensitivity. Under the excitation of $632.8 \mathrm{~nm}$, the refractive index of $\mathrm{SF} 11$ prism, $\mathrm{Ag}$ film, $\mathrm{SiO}_{2}$ interlayer, h-BN, and sensing medium (deionized water) can be determined to be $1.7786,{ }^{34} 0.14372+3.8083 \mathrm{i},{ }^{35} 1.457,{ }^{36} 1.65,{ }^{37}$ and 1.333, respectively. Considering the anisotropy of h-BN film, we employed the averaged refractive index of h-BN films to calculate and study the FR sensing performance. Next, employing the transfer matrix method and Fresnel equations (ESI†), we systematically calculate the reflectance spectra and the detection sensitivity for a tiny variation in RI.
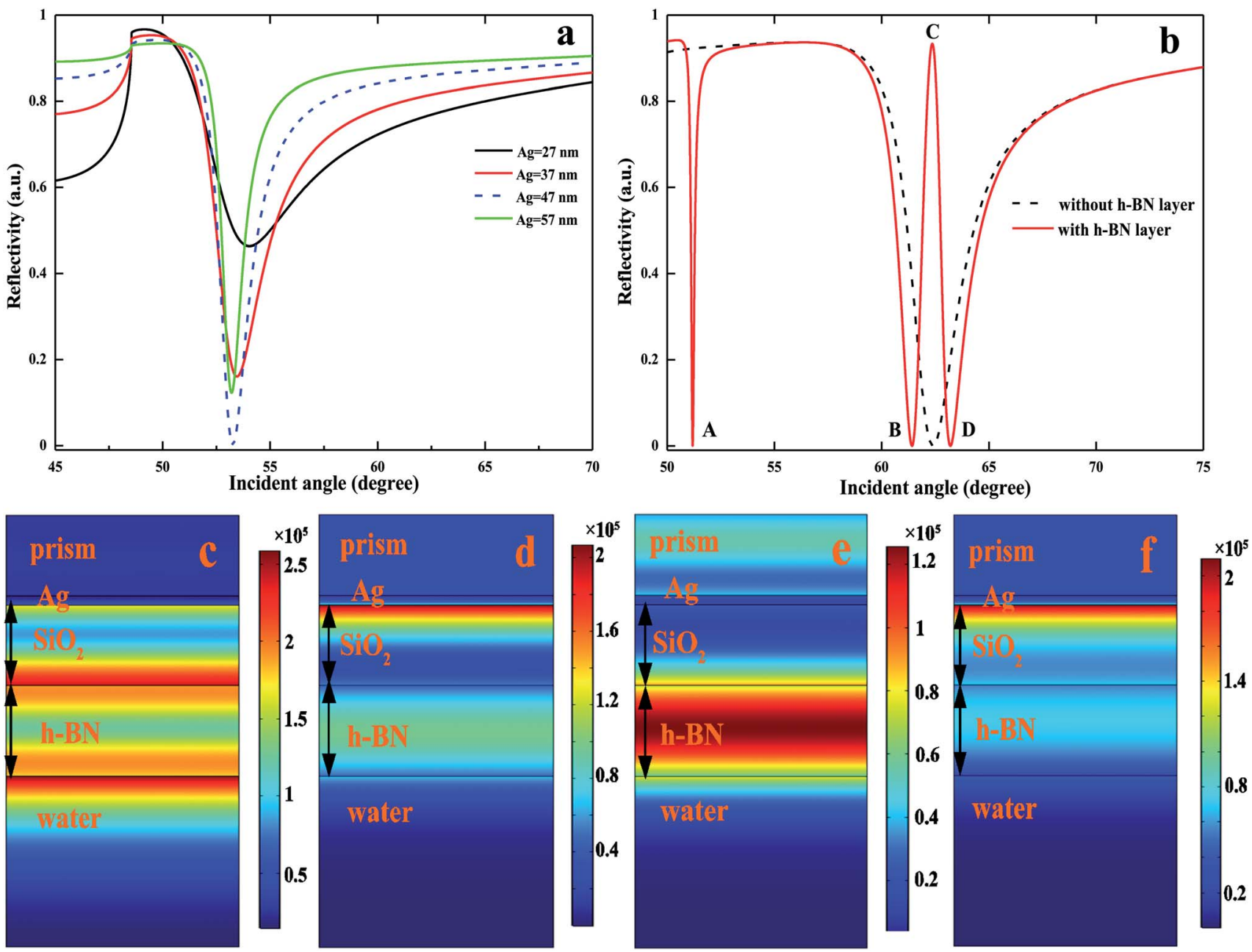

Fig. 2 (a) Calculated attenuated total reflection spectra based on a Kretschmann configuration composed of a single silver film with a thickness ranging from $27 \mathrm{~nm}$ to $57 \mathrm{~nm}$. (b) Calculated $\mathrm{FR}$ narrow lines based on a Kretschmann configuration composed of silver film/SiO 2 hybrid nanostructure by introducing the h-BN layer. (c) Calculated $|E|$ distribution corresponding to point $\mathrm{A}$ in (b); (d) calculated $|E|$ distribution corresponding to point $B$ in (b); (e) calculated $|E|$ distribution corresponding to point $C$ in (b); (f) calculated $|E|$ distribution corresponding to point $D$ in (b). The excitation wavelength is $632.8 \mathrm{~nm}$. 

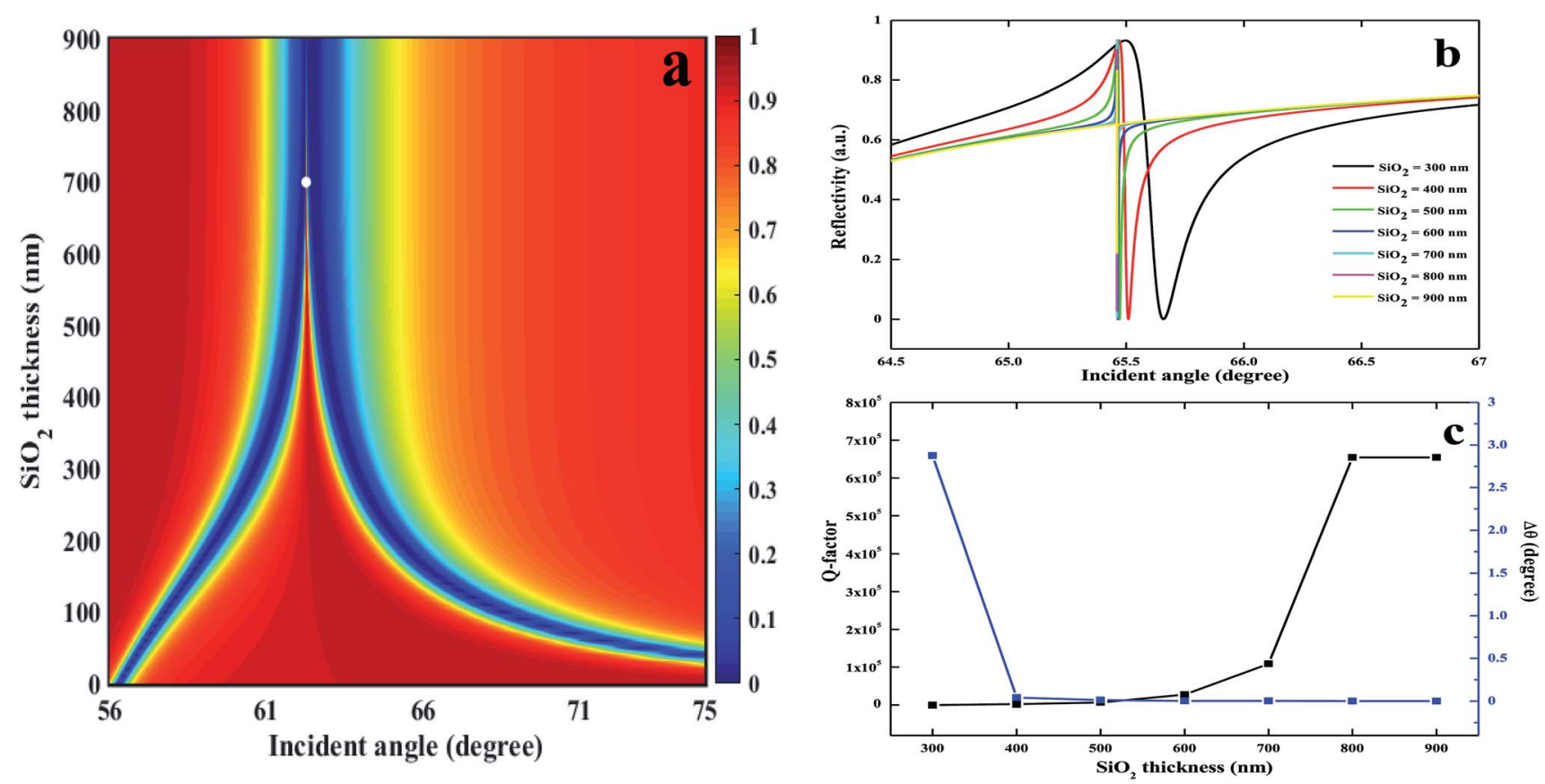

Fig. 3 (a) Contour plots of the reflection spectra as a function of incident angle and thickness of $\mathrm{SiO}_{2}$ interlayer and the thickness of silver film and h-BN are $47 \mathrm{~nm}$ and $450 \mathrm{~nm}$, respectively. (b) ATR curves with different $\mathrm{SiO}_{2}$ thickness and the thickness of silver film and h-BN are $47 \mathrm{~nm}$ and $800 \mathrm{~nm}$. (c) $Q$-Factor and the $\Delta \theta$ of the $\mathrm{FR}$ with various thicknesses of $\mathrm{SiO}_{2}$ and corresponding to (b).

\section{Results and discussion}

When the h-BN layer is absent, this hybrid nanostructure composed of silver film and $\mathrm{SiO}_{2}$ layer is a traditional SPR sensor. As shown in Fig. 2a and b, there is a significant ATR curve (black dotted curve) with a minimum reflectivity as low as $2.28 \times 10^{-4}$ at an incident angle of $62.4^{\circ}$ when the thickness of silver film and $\mathrm{SiO}_{2}$ layer are $47 \mathrm{~nm}$ and $400 \mathrm{~nm}$, respectively. It can be shown that, the SPPs mode is effectively excited at the $\mathrm{Ag}-\mathrm{SiO}_{2}$ interface, while the FR cannot be generated in absence of PWG waveguide. However, the narrow FR lines were generated when the $\mathrm{h}$-BN nanosheets with a thickness of $450 \mathrm{~nm}$ was stacked onto $400 \mathrm{~nm} \mathrm{SiO}{ }_{2}$ film. Owing to the efficient excitation of PWG pattern, both a sharp band with high absorption (point A, Fig. 2b) and a sharp peak (point C, Fig. 2b) with high reflectivity of 0.934 were obtained at $51.1^{\circ}$, and $62.4^{\circ}$, respectively. In addition, the original ATR band provided by the silver film-silicon hybrid nanostructures has been divided into two bands, namely point $\mathrm{B}$, and point $\mathrm{D}$.

To further understand the characteristics of the four resonance bands in Fig. 2b, we calculated the electric-field $|E|$ distributions based on our proposed configuration, as shown in
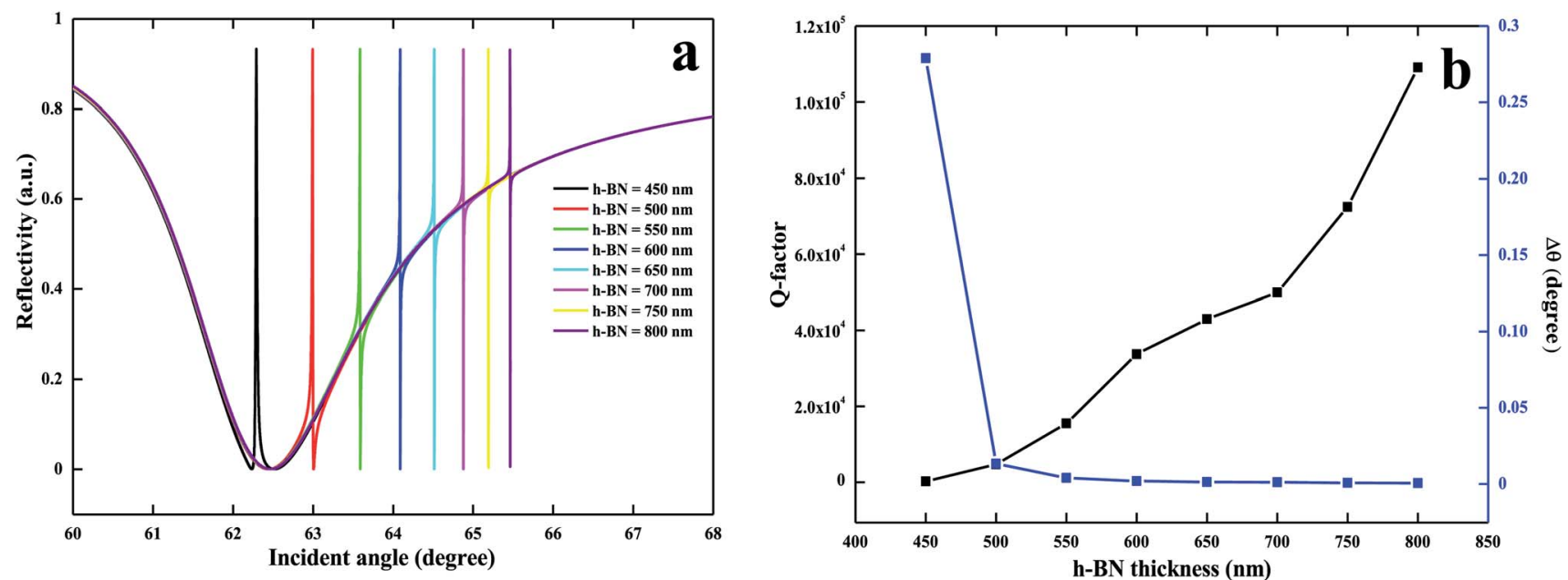

Fig. 4 (a) Calculated FR lines with various thickness of $\mathrm{h}-\mathrm{BN}$ from $450 \mathrm{~nm}$ to $800 \mathrm{~nm}$. (b) Q-Factor and the $\Delta \theta$ of the FR with different $\mathrm{h}$-BN thickness. Here, the thickness of silver film and $\mathrm{SiO}_{2}$ layer are $47 \mathrm{~nm}$ and $700 \mathrm{~nm}$, respectively. The excitation wavelength is $632.8 \mathrm{~nm}$. 
Fig. 2c-f. One can see that, when the incident angle is at $51.1^{\circ}$ (corresponding to point A), the coupling between the SPPs mode and the PWG mode is not fully achieved, as shown in Fig. 2c. In addition, for the point $B$ and point D, Fig. $2 \mathrm{~d}$ and $\mathrm{f}$ show that the SPPs mode and PWG modes cannot couple with each other, resulting in two hybrid modes at $61.4^{\circ}$ and $63.2^{\circ}$, respectively. However, Fig. 2e clearly shows that the SPPs mode and PWG mode are completely coupled at an incident angle of $62.4^{\circ}$ (point $\mathrm{C}$ ). It is worth noting that, the electric intensity corresponding to point $\mathrm{A}$ is larger than that of point $\mathrm{C}$. The reason is that, the FR line corresponding to point $\mathrm{C}$ is closer to the broad SPR band than point A. Only the PWG mode (dark mode) from the h-BN layer was successfully observed, while the SPPs mode from silver film-silicon hybrid nanostructures (bright mode) is strongly suppressed, due to the destructive interference of the two pathways. Our observations are very similar to the electromagnetically induced transparency (EIT) mechanism in an atom system, ${ }^{38}$ as well as the destructive interference of two pathways could cancel the bright mode. In our proposed configuration, there are also two pathways: (1) the incident light $\rightarrow$ SPPs mode; (2) the incident light $\rightarrow$ SPPs mode $\rightarrow$ PWG mode $\rightarrow$ SPPs mode.

Next, we systematically studied how the thickness of $\mathrm{SiO}_{2}$ interlayer influences the FR lines, as shown in Fig. 3a and b. Prior to the calculation, the thickness of h-BN was set to be $450 \mathrm{~nm}$. Fig. 3a shows the calculated map of reflection spectra when the thickness of $\mathrm{SiO}_{2}$ interlayer varied from 0 to $900 \mathrm{~nm}$. It can be found that, the $\mathrm{SiO}_{2}$ layer can serve as a coupling layer, and the coupling strength between SPPs mode and PWG mode is highly related with its thickness. It is worth noting that, the obtained typical FR shape based on our proposed configuration has a resonant peak and a resonant dip locating at the low incident angle and high incident angle sides, respectively. As the thickness of $\mathrm{SiO}_{2}$ layer increases, the narrow FR resonant peak is closer towards the broad SPP resonance band, resulting in weaker coupling between the bright mode and the dark mode. Typically, when the thickness of $\mathrm{SiO}_{2}$ layer is larger than $700 \mathrm{~nm}$, the FR line will become weak. The reason is that, as the thickness of $\mathrm{SiO}_{2}$ layer increases, the tail of SPPs is gradually far from the PWG mode, resulting in weak coupling strength between SPPs mode and PWG mode. In order to evaluate the coupling strength, we introduce a parameter $Q$-factor, which can be defined as $\theta / \Delta \theta$, where $\theta$ stands for the resonant angle of FR line, and $\Delta \theta$ denotes the difference between the FR peak and dip. As shown in Fig. 3c, for a typical FR, the thicker the thickness of $\mathrm{SiO}_{2}$ layer, the stronger the coupling strength; the resonance becomes narrower, resulting in a higher $Q$-factor.

In addition, it also can be found that, the thickness of h-BN layer can determine the position of FR line, as shown in Fig. 4a. When the thickness of $\mathrm{SiO}_{2}$ layer was set to be $700 \mathrm{~nm}$, the sharp asymmetrical FR line gradually moves towards the largeincident direction as the h-BN thickness was tuned from $450 \mathrm{~nm}$ to $800 \mathrm{~nm}$. The reason is that, the thicker the h-BN layer, the higher the refractive index of PWG mode. Thus, it needs to larger incident angle to satisfy the coupling condition. In addition, Fig. $4 \mathrm{~b}$ also shows that, the thicker the h-BN layer, the smaller the $\Delta \theta$, and the higher the $Q$-factor.

To study the sensing performance based on our proposed FR configuration, we calculated both phase and intensity detection sensitivity for a tiny variation in refractive index of sensing interface from 1.333 to 1.3331 , as shown in Fig. 5. The thickness of silver film, $\mathrm{SiO}_{2}$ layer, and h-BN nanosheets were assumed to be $47 \mathrm{~nm}, 700 \mathrm{~nm}$, and $800 \mathrm{~nm}$, respectively. The calculated results show that, the higher RI, the position of FR resonance moves towards larger incident angle. Fig. 5a shows the reflectivity change for a variation in refractive index. For intensity modulation, the optimal position locates at the sharp jump point between resonance peak and resonance dip. There is a maximum difference up to 0.33624 (the inset in Fig. $5 \mathrm{a}, \Delta R=$ $0.33624, \Delta R$ represents a change in reflectivity for a tiny
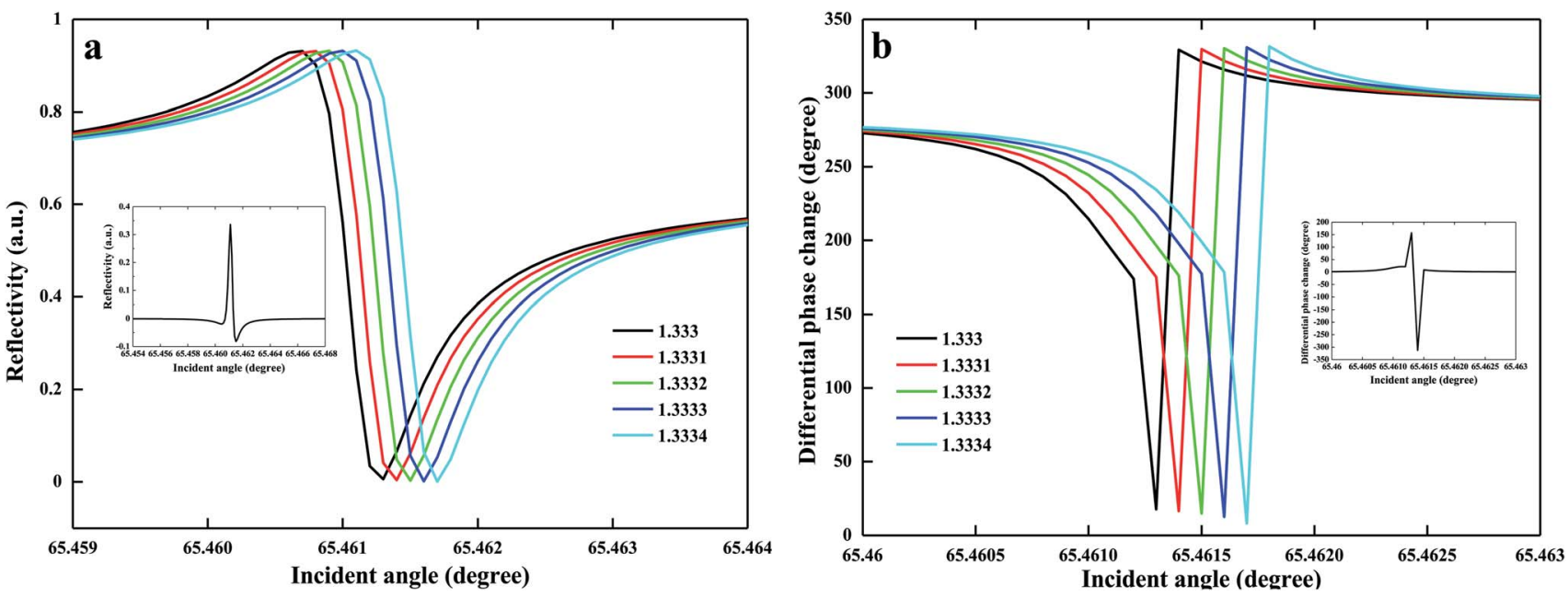

Fig. 5 (a) Variation in reflectivity with respect to the refractive index of sensing interface from 1.333 to 1.3331 . Inset shows the differences in the resonance curve before (1.333) and after (1.3331). (b) Variation in differential phase with respect to the refractive index of sensing interface from 1.333 to 1.3331 . Inset shows the differences in the resonance curve before (1.333) and after (1.3331). 
variation in RI as low as $0.0001 \mathrm{RIU}$ ) when the incident angle is $65.4611^{\circ}$. Thus, our proposed FR configuration can provide an intensity sensitivity of $3.36 \times 10^{3}$ per RIU, superior to conventional intensity-modulation SPR sensors. However, when our proposed configuration integrated with phase modulation method, it needs to introduce another s-polarized light beam, which can interfere with the p-polarized light beam employed for exciting FR. As only p-polarized light has phase jump, the differential phase of FR can be extracted from the interference pattern between p-polarized light and s-polarized light. As shown in Fig. 5b, the variation in differential phase can be modulated by varying the RI variation of sensing interface. When the incident angle is $65.4614^{\circ}$, the maximum variation in differential phase is $312.64814^{\circ}$ (the inset in Fig. 5b), providing an ultrahigh phase detection sensitivity as high as $3.13 \times 10^{6}$ degree per RIU. However, when our proposed configuration shows the largest differential phase, the obtained high reflectivity of p-polarized light is 0.0657 , which is still much higher than that of phase-modulation SPR sensor (silver film/ $\mathrm{SiO}_{2}$ :
0.00022). Limited by its low reflectivity, conventional phasemodulation SPR sensor needs to tune the incident angle of $0.001^{\circ}$ deviating from the optimal resonance angle for obtaining the p-polarized light signal. However, benefiting from its high reflectivity, our proposed configuration needn't change the optimal position of incident angle. For the SPR sensor consisting of $47 \mathrm{~nm} \mathrm{Ag}$ thin film/ $/ \mathrm{SiO}_{2}$ layer, it can produce a maximum differential phase variation up to $313.74675^{\circ}$ for a change in RI of 0.01 , and the phase detection sensitivity is 3.14 $\times 10^{4}$ degree per RIU. Therefore, our proposed configuration can achieve an EF of 100 compared to conventional phasemodulation SPR sensor. Moreover, the phase-modulation SPR sensor can offer a detectable limit of 0.001 degrees, ${ }^{39}$ while our proposed FR configuration can provide an ultralow detection limit of $10^{-9}$ RIU, resulting in ultrahigh detection accuracy and resolution.

Finally, owing to the addition of h-BN layer, the obtained ATR spectrum in Fig. 2b shows two individual resonance bands, indicating that there are the characteristics of multiple-order
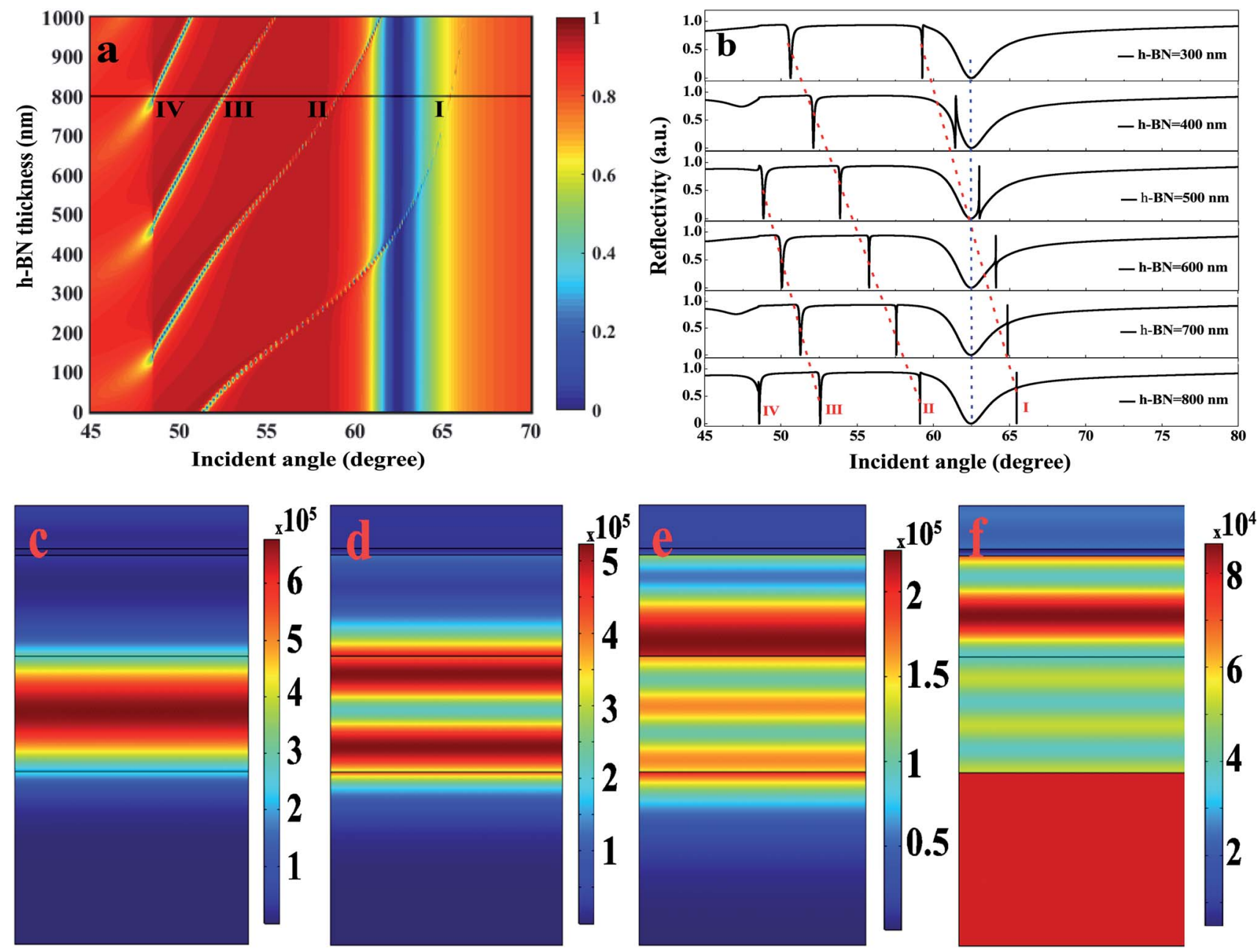

Fig. 6 (a) Map of reflection spectra as a function of incident angle when the thickness of $\mathrm{h}$-BN layer ranges from 0 to $1000 \mathrm{~nm}$. The dashed red lines denote the position of multiple FR lines, and the dashed blue line stands for the position of SPR band. (b) Reflection spectra as a function of incident angle when the thickness of h-BN layer ranges from 300 to $800 \mathrm{~nm}$. The electric field distributions corresponding to I, II, III, IV in (a) are shown in (c), (d), (e), and (f), respectively. The excitation wavelength is $632.8 \mathrm{~nm}$, and the thickness of $\mathrm{SiO}_{2}$ layer is fixed to $700 \mathrm{~nm}$. 
Fano resonances in our proposed configuration. To further exploit the coupling mechanism, we systematically studied the ATR spectra by varying the thickness of h-BN ranging from 0$1000 \mathrm{~nm}$, as shown in Fig. 6. When the thickness of silver film and $\mathrm{SiO}_{2}$ layer were fixed to $47 \mathrm{~nm}$ and $700 \mathrm{~nm}$, Fig. 6a and $\mathrm{b}$ show that, the Fano resonances have a significant red-shift and the number of Fano resonances increases with the change of the h-BN thickness (300-800 nm). Typically, there are four Fano resonances (I, II, III, IV) when the thickness of h-BN is set to be $800 \mathrm{~nm}$. It can be assumed that, the obtained multipleorder Fano resonances depends on the partial coupling between SPPs mode and the different-order PWG modes. To confirm our proposal, the distributions of electric field were calculated, as shown in Fig. 6c-f. The four Fano resonances (I, II, III, IV) correspond to first-order, second-order, third-order, and fourthorder, respectively. Fig. 6c shows that the electric field distribution based on the first-order Fano resonance is completely concentrated within the h-BN layer and has no field nodes, due to the complete coupling between the SPPs mode and the $\mathrm{TM}_{0}$ PWG mode. Noting that, the number 0 in $\mathrm{TM}_{0}$ PWG mode denotes that there is no field node. In addition, Fig. 6d shows that the electric field distribution based on second-order Fano resonance is concentrated in both the $\mathrm{SiO}_{2}-\mathrm{h}-\mathrm{BN}$ and h-BNsensing medium layer interface and has one field nodes, indicating that the SPPs mode and the $\mathrm{TM}_{1}$ PWG mode coupled completely. For the third-, fourth-order Fano resonances, Fig. 6e and $f$ show that the electric field distributions are concentrated at $\mathrm{Ag}-\mathrm{SiO}_{2}$ interface, $\mathrm{Ag}$ film layer, respectively. As the PWG modes $\left(\mathrm{TM}_{2}, \mathrm{TM}_{3}\right)$ cannot be completely coupled with SPPs mode, there are two and three field nodes, respectively. Therefore, the characteristics of multi-order Fano resonances in our proposed configuration can pave the way for designing high sensitivity sensors based on the strong FR coupling.

\section{Conclusions}

This paper proposed a phase-modulation FR biosensor by stacking 2D material h-BN nanosheets onto the silver-SiO ${ }_{2}$ interface. Owing to its characteristic of ultralow-loss of SPPs, the h-BN nanosheets act as a guiding layer and provide PWG mode. In addition, the h-BN nanosheets also can capture aromatic biochemical molecules by dipole-dipole adsorption force, which can significantly develop the absorption efficiency. The calculated results showed that, the introduction of h-BN nanosheets can produce multiple-order Fano resonances and PIT, depending on the coupling strength between SPPs mode and PWG mode. Unlike conventional phase-modulation SPR sensors, our proposed configuration can produce high reflectivity of 0.934 , which is high desirable for further phase extraction. More importantly, phase modulation of our proposed FR configuration is significantly more sensitive than intensity modulation, and a promising detection sensitivity can be obtained as high as $3.13 \times 10^{6}$ degree per RIU. Based on the FR coupling mode, our proposed configuration can be a promising candidate for exploiting novel plasmonic sensors with high sensitivity and resolution for practical applications.

\section{Conflicts of interest}

There are no conflicts of interest to declare.

\section{Acknowledgements}

This work has been partially supported by the National Key R\&D Program of China (2018YFC0910600); National Natural Science Foundation of China (61605121/61835009/61775145/61525503/ 61620106016/81727804/31771584); Project of Department of Education of Guangdong Province (2015KGJHZ002/ 2016KCXTD007); Guangdong Natural Science Foundation Innovation Team (2014A030312008); Shenzhen Basic Research Project (JCYJ20170302142902581/JCYJ20170412110212234/ JCYJ20170412105003520/JCYJ20160328144746940).

\section{References}

1 J. Homola, Surface Plasmon Resonance Sensors for Detection of Chemical and Biological Species, Chem. Rev., 2008, 108, 462-493.

2 J. Homola, S. S. Yee and G. Gauglitz, Surface plasmon resonance sensors: review, Sens. Actuators, B, 1999, 54, 3-15.

3 S. W. Zeng, D. Baillargeat, H. P. Ho and K. T. Yong, Nanomaterials enhanced surface plasmon resonance for biological and chemical sensing applications, Chem. Soc. Rev., 2014, 43, 3426-3452.

4 J. M. Brockman, B. P. Nelson and R. M. Corn, Surface Plasmon Resonance Imaging Measurements of Ultrathin Organic Films, Annu. Rev. Phys. Chem., 2000, 51, 41-63.

5 Y. H. Huang, H. P. Ho, S. Y. Wu and S. K. Kong, Detecting Phase Shifts in Surface Plasmon Resonance: A Review, Advances in Optical Technologies, 2012, 2012, 12.

6 Y. F. Yuan, X. T. Yu, Q. L. Ouyang, Y. H. Shao, J. Song, J. L. Qu, et al., Highly anisotropic black phosphorousgraphene hybrid architecture for ultrassensitive plasmonic biosensing: Theoretical insight, 2D Mater., 2018, 5, 025015.

7 L. Wu, H. S. Chu, W. S. Koh and E. P. Li, Highly sensitive graphene biosensors based on surface plasmon resonance, Opt. Express, 2010, 18, 14395-14400.

8 Y. J. Zeng, R. Hu, L. Wang, D. Y. Gu, J. N. He, S. Y. Wu, et al., Recent advances in surface plasmon resonance imaging: detection speed, sensitivity, and portability, Nanophotonics, 2017, 6, 1017-1030.

9 B. Ran and S. G. Lipson, Comparison between sensitivities of phase and intensity detection in surface plasmon resonance, Opt. Express, 2006, 14, 5641-5650.

10 S. W. Zeng, K. V. Sreekanth, J. Z. Shang, T. Yu, C. K. Chen, F. Yin, et al., Graphene-Gold Metasurface Architectures for Ultrasensitive Plasmonic Biosensing, Adv. Mater., 2015, 27, 6163-6169.

11 B. H. Ong, X. C. Yuan, S. C. Tjin, J. W. Zhang and H. M. Ng, Optimised film thickness for maximum evanescent field enhancement of a bimetallic film surface plasmon resonance biosensor, Sens. Actuators, B, 2006, 114, 10281034. 
12 B. Luk'yanchuk, N. I. Zheludev, S. A. Maier, N. J. Halas, P. Nordlander, H. Giessen, et al., The Fano resonance in plasmonic nanostructures and metamaterials, Nat. Mater., 2010, 9, 707-715.

13 A. E. Miroshnichenko, S. Flach and Y. S. Kivshar, Fano resonances in nanoscale structures, Rev. Mod. Phys., 2010, 82, 2257-2298.

14 M. F. Limonov, M. V. Rybin, A. N. Poddubny and Y. S. Kivshar, Fano resonances in photonics, Nat. Photonics, 2017, 11, 543-554.

15 S. Hayashi, D. V. Nesterenko and Z. Sekkat, Fano resonance and plasmon-induced transparency in waveguide-coupled surface plasmon resonance sensors, Appl. Phys. Express, 2015, 8, 022201.

16 S. Hayashi, D. V. Nesterenko and Z. Sekkat, Waveguidecoupled surface plasmon resonance sensor structures: Fano lineshape engineering for ultrahigh-resolution sensing, J. Phys. D: Appl. Phys., 2015, 48, 325303.

17 T. Y. Huang, S. W. Zeng, X. Zhao, Z. Cheng and P. P. Shum, Fano Resonance Enhanced Surface Plasmon Resonance Sensors Operating in Near-Infrared, Photonics, 2018, 5, 23.

18 G. G. Zheng, X. J. Zou, Y. Y. Chen, L. H. Xu and W. F. Rao, Fano resonance in graphene-MoS2 heterostructure-based surface plasmon resonance biosensor and its potential applications, Opt. Mater., 2017, 66, 171-178.

19 B. X. Ruan, Q. You, J. Q. Zhu, L. M. Wu, J. Guo, X. Y. Dai, et al., Fano resonance in double waveguides with graphene for ultrasensitive biosensor, Opt. Express, 2018, 26, 1688416892.

20 Y. Y. Liu, S. Bhowmick and B. I. Yakobson, BN White Graphene with "Colorful" Edges: The Energies and Morphology, Nano Lett., 2011, 11, 3113-3116.

21 Y. Kubota, K. Watanabe, O. Tsuda and T. Taniguchi, Deep Ultraviolet Light-Emitting Hexagonal Boron Nitride Synthesized at Atmospheric Pressure, Science, 2007, 317, 932.

22 A. J. Giles, S. Y. Dai, I. Vurgaftman, T. H. Man, S. Liu, L. Lindsay, et al., Ultralow-loss polaritons in isotopically pure boron nitride, Nat. Mater., 2018, 17, 134-139.

23 H. L. Xu, X. Wang, X. Jiang, X. Y. Dai and Y. J. Xiang, Guiding characteristics of guided waves in slab waveguide with hexagonal boron nitride, J. Appl. Phys., 2017, 122, 033103.

24 X. Ling, W. J. Fang, Y. H. Lee, P. T. Araujo, X. Zhang, J. F. Rodriguez-Nieva, et al., Raman Enhancement Effect on Two-Dimensional Layered Materials: Graphene, h-BN and $\mathrm{MoS}_{2}$, Nano Lett., 2014, 14, 3033-3040.

25 Q. Weng, X. Wang, X. Wang, Y. Bando and D. Golberg, Functionalized hexagonal boron nitride nanomaterials: emerging properties and applications, Chem. Soc. Rev., 2016, 45, 3989-4012.

26 J. Sun, C. Lu, Y. Song, Q. Ji, X. Song, Q. Li, et al., Recent progress in the tailored growth of two-dimensional hexagonal boron nitride via chemical vapour deposition, Chem. Soc. Rev., 2018, 47, 4242-4257.

27 S.-Y. Lee, T.-Y. Jeong, S. Jung and K.-J. Yee, Refractive Index Dispersion of Hexagonal Boron Nitride in the Visible and Near-Infrared, Phys. Status Solidi B, 2019, 256, 1800417.

28 F. Hui, M. A. Villena, W. J. Fang, A. Y. Lu, J. Kong, Y. Y. Shi, et al., Synthesis of large-area multilayer hexagonal boron nitride sheets on iron substrates and its use in resistive switching devices, 2D Mater., 2018, 5, 031011.

29 Y. Xu, N. Premkumar, Y. Yang and B. A. Lail, Hybrid surface phononic waveguide using hyperbolic boron nitride, opt. Express, 2016, 24, 17183-17192.

30 J. Boudiombo, J. C. Loulergue, A. Bath and P. Thevenin, Electro-optical characterization of h-BN thin film waveguides by prism coupling technique, Mater. Sci. Eng., $B$, 1999, 59, 244-247.

31 H. Xu, X. Wang, X. Jiang, X. Dai and Y. Xiang, Guiding characteristics of guided waves in slab waveguide with hexagonal boron nitride, J. Appl. Phys., 2017, 122, 033103.

32 S. Kim, J. E. Fröch, J. Christian, M. Straw, J. Bishop, D. Totonjian, et al., Photonic crystal cavities from hexagonal boron nitride, Nat. Commun., 2018, 9, 2623.

33 M. Schubert, B. Rheinländer, E. Franke, H. Neumann, J. Hahn, M. Röder, et al., Anisotropy of boron nitride thinfilm reflectivity spectra by generalized ellipsometry, Appl. Phys. Lett., 1997, 70, 1819-1821.

34 M. N. Polyanskiy, Refractive index database, https:// refractiveindexinfo.

35 A. D. Rakić, A. B. Djurišić, J. M. Elazar and M. L. Majewski, Optical properties of metallic films for vertical-cavity optoelectronic devices, Appl. Opt., 1998, 37, 5271-5283.

36 C. Z. Tan, Determination of refractive index of silica glass for infrared wavelengths by IR spectroscopy, J. Non-Cryst. Solids, 1998, 223, 158-163.

37 T. Takahashi, H. Itoh and M. Kuroda, Structure and properties of CVD-BN thick film prepared on carbon steel substrate, J. Cryst. Growth, 1981, 53, 418-422.

38 K. J. Boller, A. Imamoğlu and S. E. Harris, Observation of electromagnetically induced transparency, Phys. Rev. Lett., 1991, 66, 2593-2596.

39 A. V. Kabashin, P. Sergiy and A. N. Grigorenko, Phase and amplitude sensitivities in surface plasmon resonance bio and chemical sensing, Opt. Express, 2009, 17, 21191. 